\title{
Cauer Circuit Representation of the Homogenized Eddy-Current Field Based on the Legendre Expansion for a Magnetic Sheet
}

\section{AUTHOR(S):}

Shindo, Yuji; Miyazaki, Tatsuya; Matsuo, Tetsuji

\section{CITATION:}

Shindo, Yuji ... [et al]. Cauer Circuit Representation of the Homogenized Eddy-Current Field Based on the Legendre Expansion for a Magnetic Sheet. IEEE Transactions on Magnetics 2016, 52(3): 6300504.

ISSUE DATE:

2016-03-01

URL:

http://hdl.handle.net/2433/217021

\section{RIGHT:}

(C) 2015 IEEE. Personal use of this material is permitted. Permission from IEEE must be obtained for all other uses, in any current or future media, including reprinting/republishing this material for advertising or promotional purposes, creating new collective works, for resale or redistribution to servers or lists, or reuse of any copyrighted component of this work in other works. 


\title{
Cauer Circuit Representation of the Homogenized Eddy-Current Field Based on the Legendre Expansion for a Magnetic Sheet
}

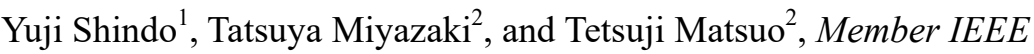 \\ ${ }^{1}$ Kawasaki Heavy Industries, Ltd., Akashi, 673-8666, Japan, shindo_yuji@khi.co.jp \\ ${ }^{2}$ Graduate School of Engineering, Kyoto University, 615-8510, Japan, matsuo.tetsuji.5u@kyoto-u.ac.jp
}

\begin{abstract}
Using the Legendre expansion of the magnetic field distribution, we derive the standard Cauer circuit representation of the frequency-dependent properties of magnetic sheets and discuss its physical meaning. The representation of nonlinear inductors is derived so as to apply the Cauer circuit in the dynamic hysteresis modeling of silicon steel. This circuit accurately reconstructs the hysteretic property under pulse-width-modulation excitation using only one or two hysteretic elements.
\end{abstract}

Index Terms-Cauer realization, dynamic hysteresis, Legendre polynomial, pulse width modulation.

\section{INTRODUCTION}

$\mathrm{P}$ ROGRESS in the semiconductor technology has resulted in advanced power control with high-frequency switching operations that induce complex dynamic hysteretic magnetic fields in iron cores. Minor hysteresis loops and eddy-current fields with thin skin depths are significant within these cores.

Several homogenization methods [1]-[5] have been developed for efficient analysis of the laminated cores that avoid the finite-element division along the stacking direction of the silicon steel sheets. However, an accurate evaluation of the eddy-current field in sheets displaying nonlinear magnetic properties is difficult without a one-dimensional sub-analysis along the sheet-thickness direction [1]-[4]. The sub-analysis requires the division along the thickness direction to skin depth scale. For example, if the skin depth is $0.5 \mathrm{~mm}$ at $50 \mathrm{~Hz}$, it becomes 0.05 and $0.01 \mathrm{~mm}$ at $5 \mathrm{kHz}$ and $125 \mathrm{kHz}$, respectively, resulting in a fine element grid for the steel sheet.

An efficient representation of the frequency-dependent properties of magnetic sheets is achieved using the standard and physical Cauer circuit representations [5]-[7] based on the linear eddy-current theory. Although the standard Cauer circuit is obtained directly from the linear theory, it has not been applied to nonlinear eddy-current analysis because its physical meaning is unclear. In contrast, the physical Cauer circuit has been used in nonlinear analysis because of its clear physical meaning. In the nonlinear case, however, the physical Cauer circuit requires more inductive elements than expected from the linear circuit, even after circuit optimization [5].

Ref. [8] discusses the meaning of the truncated standard Cauer circuit with two inductors comparing the homogenization method developed in Ref. [2], [3] based on the Legendre expansion. Here we derive the standard Cauer circuit directly from the Legendre expansion for the magnetic field to consider the physical meaning of this circuit. The

Manuscript received April 1, 2015; revised May 15, 2015 and June 1, 2015; accepted July 1, 2015. Date of publication July 10, 2015; date of current version July 31, 2015. Corresponding author: T. Matsuo (e-mail: matsuo.tetsuji.5u@kyoto-u.ac.jp).

Color versions of one or more of the figures in this paper are available online at http://ieeexplore.ieee.org.

Digital Object Identifier (inserted by IEEE) representation of a nonlinear inductor is derived to apply the standard Cauer circuit to the dynamic hysteresis modeling of a steel sheet.

\section{Derivation of CAuer Circuit By Legendre EXPANSION}

\section{A. Cauer realization}

The magnetic field in the steel sheet of thickness $d$ is governed by

$$
\frac{\partial^{2} H}{\partial z^{2}}=\sigma \frac{\partial B}{\partial t}, \quad\left(-\frac{d}{2} \leq z \leq \frac{d}{2}\right)
$$

where $\sigma$ is the conductivity. A linear eddy-current theory for the magnetic sheet gives the relationship between the average magnetic flux density $B_{\mathrm{av}}$ and the surface magnetic field $H_{\mathrm{s}}$ as

$$
\frac{B_{\mathrm{av}}}{H_{\mathrm{s}}}=\mu \frac{2}{k d} \tan \left(\frac{k d}{2}\right)=\mu \frac{2}{\mathrm{j} k d} \tanh \left(\frac{\mathrm{j} k d}{2}\right),
$$

where $k=(-j \omega \sigma \mu)^{1 / 2}, \omega$ is the angular frequency, and $\mu$ the permeability. By expanding $\tan (k d / 2)$ or $\tanh (\mathrm{j} k d / 2)$, (2) is represented by the infinite $R L$ ladder circuit [6], [7] (see Fig. $1)$, where $\mu$ and $4 / \sigma d^{2}$ are replaced by the inductance $L$ and resistance $R$, respectively. This circuit is called the standard Cauer circuit.

\section{B. Legendre expansion}

Refs. [2], [3] describes a homogenization method where the magnetic flux density distribution along the thickness direction is expanded as Legendre polynomials [9] $P_{2 n}(x)(-1$ $\leq x \leq 1, n=0,1, \ldots)$ as

$$
B(t, z)=b_{0}(t) P_{0}\left(\frac{2 z}{d}\right)+b_{2}(t) P_{2}\left(\frac{2 z}{d}\right)+b_{4}(t) P_{4}\left(\frac{2 z}{d}\right)+\cdots
$$

This subsection derives the standard Cauer circuit using (3) and the orthogonality of the Legendre polynomials below:

$$
\int_{-1}^{1} P_{m}(x) P_{n}(x) \mathrm{d} x=\left\{\begin{array}{cc}
0 & (m \neq n) \\
2 /(2 m+1) & (m=n)
\end{array} .\right.
$$

By defining

$$
\boldsymbol{P}=\left[P_{0}\left(\frac{2 z}{d}\right), P_{2}\left(\frac{2 z}{d}\right), P_{4}\left(\frac{2 z}{d}\right), \cdots\right]^{\mathrm{T}},
$$




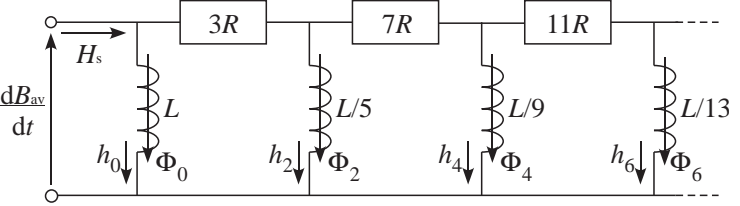

Fig. 1. Standard Cauer circuit.
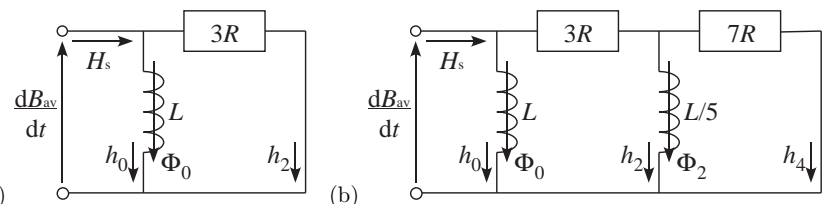

Fig. 2. Truncated standard Cauer circuit: (a) with one inductor and (b) with two inductors.
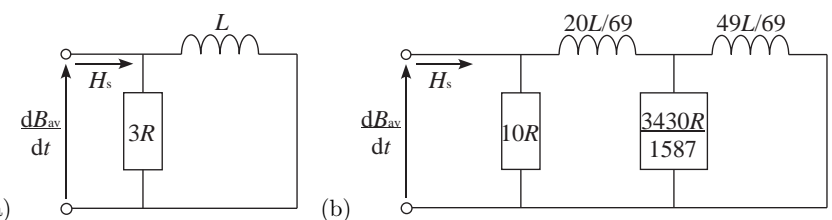

Fig. 3. Physical Cauer circuit: (a) with one inductor and (b) with two inductors

$$
\boldsymbol{h}=\left[h_{0}(t), h_{2}(t), h_{4}(t), \cdots\right]^{\mathrm{T}}=\frac{1}{\mu}\left[b_{0}(t), b_{2}(t), b_{4}(t), \cdots\right]^{\mathrm{T}},
$$

(1) is rewritten as

$$
\boldsymbol{h}^{\mathrm{T}} \frac{\partial^{2} \boldsymbol{P}}{\partial z^{2}}=\sigma \frac{\mathrm{d}\left(\mu \boldsymbol{h}^{\mathrm{T}}\right)}{\mathrm{d} t} \boldsymbol{P} .
$$

Using the relations,

$$
\begin{aligned}
& \frac{\mathrm{d} P_{2 n}(x)}{\mathrm{d} x}=(4 n-1) P_{2 n-1}(x)+(4 n-5) P_{2 n-3}(x)+\cdots+3 P_{1}(x), \\
& \frac{\mathrm{d} P_{2 n+1}(x)}{\mathrm{d} x}=(4 n+1) P_{2 n}(x)+(4 n-3) P_{2 n-2}(x)+\cdots+P_{0}(x),
\end{aligned}
$$

(7) is written

$$
\left[\sigma \frac{\mathrm{d}\left(\mu \boldsymbol{h}^{\mathrm{T}}\right)}{\mathrm{d} t}-\frac{4}{d^{2}} \boldsymbol{h}^{\mathrm{T}} \boldsymbol{G} \boldsymbol{F}\right] \boldsymbol{P}=0
$$

where

$$
\begin{aligned}
& \boldsymbol{F}=\operatorname{diag}(1,5,9, \cdots), \\
& \boldsymbol{G}=\left[\begin{array}{ccccc}
0 & 0 & 0 & 0 & \cdots \\
3 & 0 & 0 & 0 & \cdots \\
3+7 & 7 & 0 & 0 & \ldots \\
3+7+11 & 7+11 & 11 & 0 & \ldots \\
\vdots & \vdots & \vdots & \vdots & \ddots
\end{array}\right] .
\end{aligned}
$$

From (9) and the orthogonality (4),

$$
\boldsymbol{F}^{-1} \frac{\mathrm{d}(L \boldsymbol{h})}{\mathrm{d} t}-\frac{4}{\sigma d^{2}} \boldsymbol{G}^{\mathrm{T}} \boldsymbol{h}=0
$$

is obtained. As the surface magnetic field $H_{\mathrm{s}}$ is given as

$$
H_{\mathrm{s}}(t)=\frac{1}{\mu} B\left(t, \frac{d}{2}\right)=h_{0}(t)+h_{2}(t)+h_{4}(t)+\cdots
$$

(12) describes the equation of state for the standard Cauer circuit of Fig. 1, where

$$
\Phi_{2 n}(t)=\frac{b_{2 n}(t)}{4 n+1} .
$$

The physical meaning of the standard Cauer circuit was discussed in [8] and is given as follows. When the frequency is low, $h_{0}(t)\left(\approx H_{\mathrm{s}}(t)\right)$ is the dominant current and the flux change $\mathrm{d} \Phi_{0} / \mathrm{d} t=L \mathrm{~d} h_{0} / \mathrm{d} t$ induces an eddy-current $h_{2}(t) \approx\left(\mathrm{d} B_{\text {av }} / \mathrm{d} t\right) /(3 R)$. Accordingly, the magnetic flux $\Phi_{2}=(L / 5) h_{2}$ is regarded as the secondary flux generated by the induced current $h_{2}$. This explanation is supported by the Legendre expansion. The uniformly distributed magnetic flux density $b_{0} P_{0}$ induces the eddy current distributed linearly along the $z$-direction, which yields the parabolically distributed $b_{2} P_{2}$.

Furthermore, (3), (6), and the orthogonality (4) give the magnetic energy $w(t)$ per unit volume as

$$
\begin{aligned}
w(t) & =\frac{1}{d} \cdot \frac{1}{2 \mu} \int_{-d / 2}^{d / 2} B^{2}(t, z) \mathrm{d} z \\
& =\frac{1}{d} \cdot \frac{\mu}{2} \sum_{n=0}^{\infty} \int_{-d / 2}^{d / 2} h_{2 n}^{2}(t) P_{2 n}^{2}\left(\frac{2 z}{d}\right) \mathrm{d} z=\frac{1}{2} \cdot \sum_{n=0}^{\infty} \frac{L}{4 n+1} h_{2 n}^{2}(t)
\end{aligned}
$$

Note that the energy associated with the flux distribution $\mu h_{2 n}(t) P_{2 n}(2 z / d)$ in the magnetic sheet coincides with the energy stored in the inductor $L /(4 n+1)$ of the Cauer circuit. The physical magnetic energy in the magnetic sheet is conserved in the Cauer circuit representation.

By truncating the standard Cauer circuit (Fig. 2), they can be converted to equivalent types of $R L$ ladder circuits (Fig. 3). These circuits are called physical Cauer circuits because the ratio of the inductances corresponds to the nonuniform physical division [5]-[7] of the half thickness $d / 2$. The truncated circuit shown in Fig. 2(a) is the same as the circuit in Fig. 3(a), which in classical eddy-current theory represents

$$
H_{\mathrm{s}}(t)=\frac{1}{\mu} B_{\mathrm{av}}(t)+\frac{\sigma d^{2}}{12} \frac{\mathrm{d} B_{\mathrm{av}}}{\mathrm{d} t} .
$$

The equivalence of truncated standard Cauer circuit to the homogenization method in [2], [3] is proved in the Appendix.

When the Cauer circuit is truncated with $(N+1)$ pairs of $L /$ $(4 n+1)$ and $(4 n+3) R(n=0, \ldots, N)$, its impedance at high frequency $(\omega>>R / L)$ asymptotically becomes $3 R+7 R+\ldots+$ $(4 N+3) R=(N+1)(2 N+3) R$ because the inductors are approximately open-circuited. This means that the iron loss for the sinusoidal $B$ with amplitude $B_{\mathrm{m}}$ becomes

$$
P_{N} \approx \frac{\omega^{2} B_{m}^{2}}{2(N+1)(2 N+3) R}(\omega L>>R) .
$$

From (2), the infinite Cauer circuit gives the iron loss as

$$
P \approx \frac{\omega^{3 / 2} B_{m}^{2}}{\sqrt{8 R L}}(\omega L>>R) .
$$

Equating (17) with (18), the frequency range where the truncated Cauer circuit is applicable is evaluated as

$$
\omega<\omega_{N}=\frac{(N+1)^{2}(2 N+3)^{2} R}{2 L} .
$$

Equation (19) implies that a small increase of $N$ results in a large increase of frequency range.

\section{Nonlinear inductors}

If the static magnetic property of a steel sheet has nonlinearity represented by $H_{\mathrm{s}}=H_{\mathrm{DC}}\left(B_{\mathrm{av}}\right)$, the first inductor $L$ is replaced by relation $h_{0}=H_{\mathrm{DC}}\left(\Phi_{0}\right)$. Magnetic fluxes $\Phi_{2}, \Phi_{4}$, 
... can be regarded as corrections to flux $\Phi_{0}$. If the flux correction is small and $H_{\mathrm{DC}}$ is not a hysteretic function, one obtains

$$
\begin{aligned}
H(t, z) & =H_{\mathrm{DC}}\left(b_{0}(t)+b_{2}(t) P_{2}\left(\frac{2 z}{d}\right)+b_{4}(t) P_{4}\left(\frac{2 z}{d}\right)+\cdots\right) \\
\approx & H_{\mathrm{DC}}\left(b_{0}(t)\right)+\frac{1}{\mu_{\mathrm{d}}}\left(b_{2}(t) P_{2}\left(\frac{2 z}{d}\right)+b_{4}(t) P_{4}\left(\frac{2 z}{d}\right)+\cdots\right)
\end{aligned}
$$

where $\mu_{\mathrm{d}}=\left[\mathrm{d} H_{\mathrm{DC}}\left(\Phi_{0}\right) / \mathrm{d} \Phi_{0}\right]^{-1}$ is the differential permeability. By setting

$$
\boldsymbol{h}=\frac{1}{\mu_{\mathrm{d}}}\left[b_{0}(t), b_{2}(t), b_{4}(t), \cdots\right]^{\mathrm{T}}
$$

and replacing $\mu$ and $L$ by $\mu_{\mathrm{d}}$, (7), (9), and consequently (12) hold. By setting,

$$
h_{0}(t)=H_{\mathrm{DC}}\left(b_{0}(t)\right), h_{2 n}(t)=b_{2 n}(t) / \mu_{\mathrm{d}}(n=1,2, \ldots) .
$$

(12), (13), and (14) describes the equation of state for the standard Cauer circuit (Fig. 1) with the state variables $\Phi_{2 n}(n=$ $0,1, \ldots)$, where $L h_{0}$ is replaced by $\Phi_{0}$ satisfying $h_{0}=H_{\mathrm{DC}}\left(\Phi_{0}\right)$. The relation between $h_{2 n}$ and $\Phi_{2 n}(n=1,2, \ldots)$ is given as

$$
h_{2 n}(t)=(4 n+1) \Phi_{2 n}(t) / \mu_{\mathrm{d}},
$$

where $\mu_{\mathrm{d}}$ is a function of $b_{0}=\Phi_{0}$

If $H_{\mathrm{DC}}$ is hysteretic, however, the linear approximation (20) does not hold because the past history of the input perturbation affects the present hysteretic output. Instead of using (20), Ref. [8] proposed a finite difference approximation

$$
h_{2 n}=\frac{4 n+1}{\varepsilon}\left[H_{\mathrm{DC}}\left(\Phi_{0}+\varepsilon \Phi_{2 n}\right)-H_{\mathrm{DC}}\left(\Phi_{0}\right)\right],
$$

where $\varepsilon$ is a constant and $H_{\mathrm{DC}}$ is affected by the history of $\Phi_{0}$ and $\Phi_{0}+\varepsilon \Phi_{2 n}$.

To represent the nonlinear inductor $L /(4 n+1)(n=1,2, \ldots)$, we propose a rough approximation using (20). By neglecting hysteresis, $H_{\mathrm{DC}}$ is approximated by a single-valued function $H_{0}(B)$ to give the differential permeability as $\mu_{\mathrm{d}}=$ [d $\left.H_{0}\left(\Phi_{0}\right) / \mathrm{d} \Phi_{0}\right]^{-1}$. To approximate $H_{\mathrm{DC}}$, two types of singlevalued functions below are examined:

$$
\begin{aligned}
& H_{0}=H_{\text {ave }}(B)=\frac{1}{2}\left[H_{+}(B)+H_{-}(B)\right], \\
& H_{0}=H_{\text {rev }}(B),
\end{aligned}
$$

where $H_{\text {ave }}(B)$ is given by the average of ascending curve $H_{+}(B)$ and descending curve $H_{-}(B)$ of the major loop of $H_{\mathrm{DC}}(B)$. The second function $H_{\mathrm{rev}}(B)$ is the reversible component of $H_{\mathrm{DC}}(B)$ used to approximate $H_{\mathrm{DC}}(B)$ under the pulse-width-modulation (PWM) excitation. If the fundamental frequency of the PWM waveform is low, the fundamental component is not affected by elements of $L /(4 n+1)$ and $(4 n+3) R(n \geq 1)$. The PWM carrier frequency yields small minor hysteresis loops, which are affected by $L / 5$ and $7 R$. Accordingly, $\mu_{\mathrm{d}}$ can be given by the incremental permeability $(\Delta B / \Delta H$ in Fig. 4) of minor loops, which is roughly approximated by $\mathrm{d} H_{\text {rev }}(B) / \mathrm{d} B$.

In the physical Cauer circuit, the $k$-th inductor $L / \alpha_{k}$ can be replaced by the relation $h_{k}=H_{\mathrm{DC}}\left(\alpha_{k} \Phi_{k}\right)$ because $L / \alpha_{k}$ corresponds to the magnetic flux passing through $1 / \alpha_{k}$ of the sheet's thickness. A similar interpretation for inductor $L /(4 n+1)$ in the standard Cauer circuit is given by $h_{2 n}=H_{\mathrm{DC}}\left((4 n+1) \Phi_{2 n}\right)$, which does not give more accurate results than (24) [8].

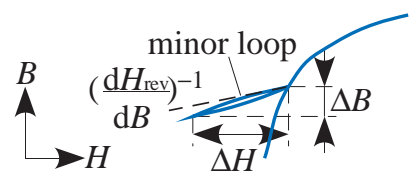

Fig. 4. Permeability for the minor B-H loop.

\section{NUMERICAL RESULT}

B-H loops and iron losses of a non-oriented silicon steel sheet, JIS: 35A300 were measured with a single sheet tester using two-types of PWM waveforms, for which the fundamental and carrier frequencies are $50 \mathrm{~Hz}$ and $10 \mathrm{kHz}$. The static hysteretic property $H=H_{\mathrm{DC}}(B)$ for the steel sheet is represented by the play model [10]. The measured $B_{\mathrm{av}}$ is given to simulate the surface field $H_{\mathrm{s}}$.
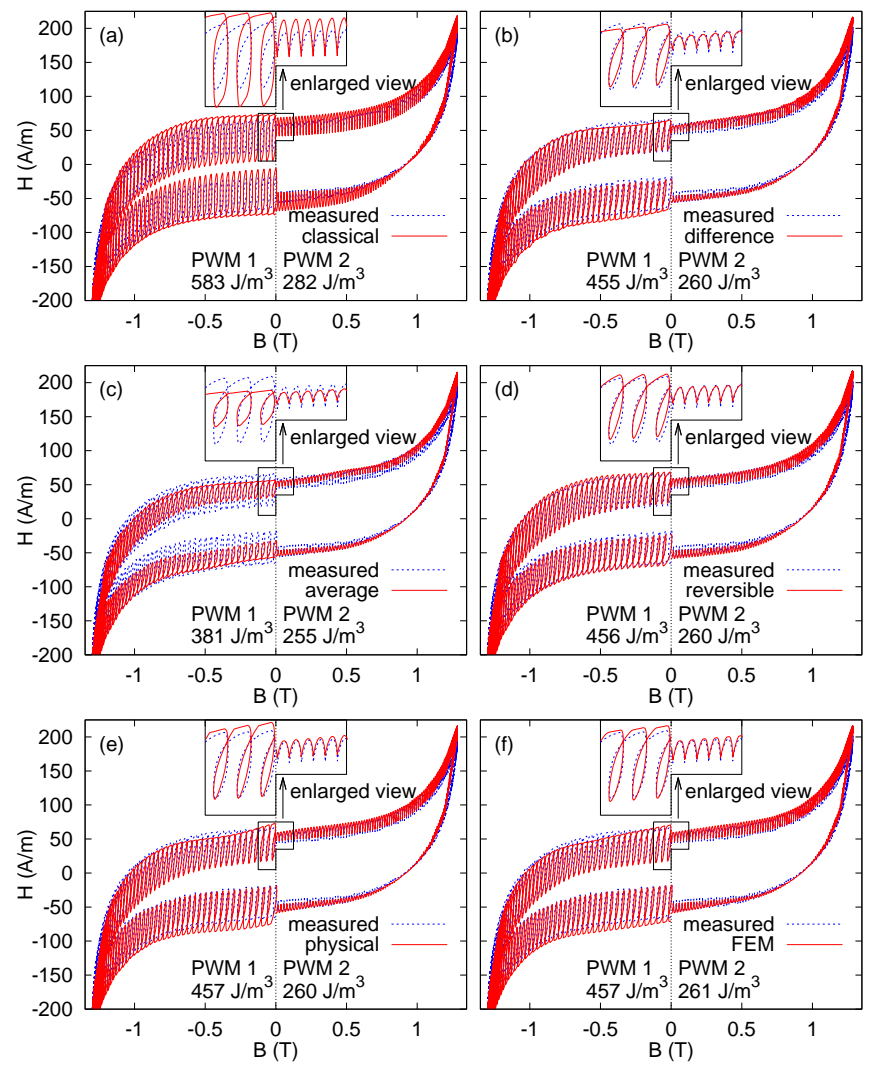

Fig. 5. Simulated B-H loops for 2 types of PWM waveforms where measured iron losses per cycle are 458 and $252 \mathrm{~J} / \mathrm{m}^{3}$ : (a) classical eddy-current theory, (b) standard Cauer circuit with finite-difference approximation (21) with $\varepsilon=1$, (c) standard Cauer circuit with average approximation, (d) standard Cauer circuit with reversible component approximation, (e) physical Cauer circuit, and (f) finite element method.

Fig. 5(a) shows the simulated B-H loops produced by the standard Cauer circuit, shown in Fig. 2(a), which is equivalent to the classical eddy-current theory. Fig. 5(b)-(d) given by the circuit shown in Fig. 2(b), where (b) the finite difference approximation (24), (c) the average approximation $H_{\text {ave }}$, and (d) the reversible component $H_{\text {rev }}$ are used to represent the second inductor $L / 5$. For comparison, Fig. 5(e) and (f) shows the $\mathrm{B}-\mathrm{H}$ loops given by the physical Cauer circuit shown in Fig. 3(b) and the one-dimensional finite element eddy-current analysis along the $z$-direction with 10 first-order elements. 
Assuming that $\Phi_{2 n}$ is small, $\varepsilon$ in (24) is set to 1. Fig. 5(a)-(f) presents the computed iron losses per cycle for the PWM waveforms 1 and 2, which in measurements yield 458 and 252 $\mathrm{J} / \mathrm{m}^{3}$. The PWM waveform 1 has $1.2 \%$ of 200 -th harmonic in $B_{\text {av }}$ due to the carrier wave whereas the waveform 2 has $0.17 \%$ of $(400 \pm 1)$-th harmonics. If the linear magnetic property is assumed with $L=5 \times 10^{-3} \mathrm{H} / \mathrm{m}$ and $R=12 \Omega / \mathrm{m}$, $\omega_{0} / 2 \pi$ and $\omega_{1} / 2 \pi$ are 1.7 and $19 \mathrm{kHz}$ from (19) and the ratio of amplitude of $b_{2}$ to that of $b_{0}$ is 0.09 and 0.06 for the waveforms 1 and 2 . The classical eddy-current theory overestimates the component of carrier frequency [Fig. 5(a)], because the effect of secondary flux $\Phi_{2}$ is neglected even though the carrier frequency is larger than $\omega_{0} / 2 \pi$. The average approximation underestimates the minor loops [Fig. 5(c)] because the inductance $L / 5$ given by $\left[\mathrm{d} H_{\text {ave }}\left(\Phi_{0}\right) / \mathrm{d} \Phi_{0}\right]^{-1} / 5$ is too large for the minor $\mathrm{B}-\mathrm{H}$ loops. In contrast, the reversible component approximation achieves an accurate representation as the finite-difference approximation and the finite-element method (FEM) because $\mathrm{d} H_{\text {rev }}\left(\Phi_{0}\right) / \mathrm{d} \Phi_{0}$ gives a good approximation for the permeability of the minor loops. Note that the standard Cauer circuit using $H_{\text {rev }}$ requires only one hysteretic inductor whereas the Cauer circuit with the finitedifference approximation needs two hysteretic inductors, and the FEM requires as many hysteretic elements as the number of finite elements. The physical Cauer circuit with two hysteretic inductors results in a slightly inaccurate outer B-Hloop representation, which is improved by increasing the number of inductors because the physical Cauer circuit is equivalent to the nonuniformly divided FEM.

\section{CONCLUSION}

Using the Legendre expansion of the magnetic field distribution, we derive the standard Cauer circuit representation of the frequency-dependent properties of magnetic sheets. The circuit representation provides us useful information such as the applicable frequency limitation due to the truncation. The representation of the nonlinear inductors is derived and gives accurate dynamic hysteretic properties for silicon steel under PWM excitation only with one or two hysteretic inductors.

\section{APPENDIX}

From (8), one obtains that

$$
\int P_{n}(x) \mathrm{d} x=\frac{1}{2 n+1}\left[P_{n+1}(x)-P_{n-1}(x)\right](n=1,2, \ldots) .
$$

From (27), we have

$$
\begin{gathered}
\iint P_{2 n}(x) \mathrm{d} x \mathrm{~d} x=\frac{1}{4 n+1}\left[\frac{P_{2 n+2}(x)-P_{2 n}(x)}{4 n+3}-\frac{P_{2 n}(x)-P_{2 n-2}(x)}{4 n-1}\right], \\
\iint P_{0}(x) \mathrm{d} x \mathrm{~d} x=\int P_{1}(x) \mathrm{d} x=\left[P_{2}(x)-P_{0}(x)\right] / 3 .
\end{gathered}
$$

Using the truncated Legendre expansion

$$
B(t, z)=\sum_{n=0}^{N} b_{2 n}(t) P_{2 n}\left(\frac{2 z}{d}\right)
$$

Ref. [2], [3] integrates (1) twice with respect to $z$ to obtain

$$
H(t, z)=\sigma \iint \frac{\mathrm{d} B(t, z)}{\mathrm{d} t} \mathrm{~d} z \mathrm{~d} z=\sigma \sum_{n=0}^{N} \frac{\mathrm{d} b_{2 n}(t)}{\mathrm{d} t} \iint P_{2 n}\left(\frac{2 z}{d}\right) \mathrm{d} z \mathrm{~d} z .
$$

Using (30), $H(t, z)$ is rewritten in the form

$$
\begin{aligned}
H(t, z) & =H_{\mathrm{s}}(t)+\frac{\sigma d^{2}}{4} \frac{\mathrm{d} b_{0}}{\mathrm{~d} t} \frac{P_{2}-P_{0}}{3} \\
& +\sum_{n=1}^{N} \frac{\sigma d^{2}}{4(4 n+1)} \frac{\mathrm{d} b_{2 n}}{\mathrm{~d} t}\left(\frac{P_{2 n+2}-P_{2 n}}{4 n+3}-\frac{P_{2 n}-P_{2 n-2}}{4 n-1}\right)
\end{aligned}
$$

If linearity is assumed for the magnetic property $H(t, z)=B(t$, $z) / \mu$, the orthogonality of Legendre functions leads to

$$
\begin{aligned}
\frac{1}{\mu} b_{2 n}(t)= & \frac{1}{4 n+3} \frac{\sigma d^{2}}{4(4 n+5)} \frac{\mathrm{d} b_{2 n+2}}{\mathrm{~d} t} \\
& -\left(\frac{1}{4 n+3}+\frac{1}{4 n-1}\right) \frac{\sigma d^{2}}{4(4 n+1)} \frac{\mathrm{d} b_{2 n}}{\mathrm{~d} t} \quad(1 \leq n \leq N) \\
& +\frac{1}{4 n-1} \frac{\sigma d^{2}}{4(4 n-3)} \frac{\mathrm{d} b_{2 n-2}}{\mathrm{~d} t} \\
\frac{1}{\mu} b_{0}(t)= & H_{\mathrm{s}}(t)+\frac{1}{3} \frac{\sigma d^{2}}{4 \cdot 5} \frac{\mathrm{d} b_{2}}{\mathrm{~d} t}-\frac{1}{3} \frac{\sigma d^{2}}{4} \frac{\mathrm{d} b_{0}}{\mathrm{~d} t}
\end{aligned}
$$

where $b_{2 N+2}$ is set to 0 . Setting as in (14), (30) is rewritten as

$$
\begin{aligned}
\frac{4 n+1}{\mu} \Phi_{2 n}(t)= & \frac{1}{(4 n+3) R}\left(\frac{\mathrm{d} \Phi_{2 n+2}}{\mathrm{~d} t}-\frac{\mathrm{d} \Phi_{2 n}}{\mathrm{~d} t}\right) \\
& +\frac{1}{(4 n-1) R}\left(\frac{\mathrm{d} \Phi_{2 n-2}}{\mathrm{~d} t}-\frac{\mathrm{d} \Phi_{2 n}}{\mathrm{~d} t}\right) \\
\frac{1}{\mu} \Phi_{0}(t)= & H_{\mathrm{s}}(t)+\frac{1}{3 R}\left(\frac{\mathrm{d} \Phi_{2}}{\mathrm{~d} t}-\frac{\mathrm{d} \Phi_{0}}{\mathrm{~d} t}\right)
\end{aligned}
$$

where $\Phi_{2 N+2}$ is set to 0 . The equation of state for the standard Cauer circuit truncated with $N$ inductors is given by (33). Using the linear approximation (20), the weak form of the constitutive equation proposed in [3] for the nonlinear property is similarly represented by the standard Cauer circuit.

\section{REFERENCES}

[1] O. Bottauscio, M. Chiampi, D. Chiarabaglio, "Advanced model of laminated magnetic cores for two-dimensional field analysis," IEEE Trans. Magn., vol. 36, pp. 561-573, May 2000.

[2] J. Gyselinck, P. Dular, "A time-domain homogenization technique for laminated iron cores in 3-D finite-element models," IEEE Trans. Magn., vol. 40, pp. 856-859, Mar. 2004.

[3] J. Gyselinck, R.V. Sabariego, P. Dular, "A nonlinear time-domain homogenization technique for laminated iron cores in three-dimensional finite-element models," IEEE Trans. Magn., vol. 42, pp. 763-766,Apr. 2006.

[4] I. Niyonzima, R.V. Sabariego, P. Dular, F. Henrotte, C. Geuzaine, "Computational homogenization for laminated ferromagnetic cores in magnetodynamics," IEEE Trans. Magn., vol. 49, pp. 2049-2052, May 2013.

[5] J.H. Krah, "Optimum discretization of a physical Cauer circuit," IEEE Trans. Magn., vol. 41, pp. 1444-1447, May 2005.

[6] E. Tarasiewicz, A.S. Morched, A. Narang, E.P. Dick, "Frequency dependent eddy current models for nonlinear iron cores," IEEE Trans. Power Syst., vol. 8, pp. 588-597, May 1993.

[7] Y. Shindo, O. Noro, "Simple circuit simulation models for eddy current in magnetic sheets and wires," IEEJ Trans. FM, vol.134, pp. 173-181, Apr. 2014.

[8] T. Miyazaki, T. Mifune, T. Matsuo, Y. Shindo, Y. Takahashi, K. Fujiwara, "Equivalent circuit modeling of dynamic hysteretic property of silicon steel sheet under pulse width modulation excitation," J. Appl. Phys. vol. 117, 17D110, 2015.

[9] W. W. Bell, Special functions for scientists and engineers, New York: Dover, 2004, pp. 42-91.

[10] T. Matsuo, M. Shimasaki, "An identification method of play model with input-dependent shape function," IEEE Trans. Magn., vol. 41, pp. 31123114 , Oct. 2005 